\title{
Unilateral Anophthalmos
}

National Cancer Institute

\section{Source}

National Cancer Institute. Unilateral Anophthalmos. NCI Thesaurus. Code C101188.

A rare congenital abnormality characterized by the complete absence of ocular tissue in one orbit. 